05

\title{
Микромагнитные структуры, индуцированные неоднородным электрическим полем, в магнитодноосных пленках с фрлексомагнитоэлектрическим эфффектом
}

\author{
(C) Р.М. Вахитов ${ }^{1}$, 3.В. Гареева ${ }^{1,2}$, Р.В. Солонецкий ${ }^{3}$, Ф.А. Мажитова ${ }^{1}$ \\ ${ }^{1}$ Башкирский государственный университет, \\ Уфа, Россия \\ ${ }^{2}$ Институт фризики молекул и кристаллов УФИЦ РАН, \\ Уфра, Россия \\ ${ }^{3}$ Уфримский государственный авиационный технический университет, \\ Уфра, Россия \\ E-mail: VakhitovRM@Yahoo.com
}

Поступила в Редакцию 27 декабря 2018 г.

В окончательной редакции 27 декабря 2018 г.

Принята к публикации 28 декабря 2018 г.

Исследуются особенности проявления флексомагнитоэлектрического эффекта в магнитоодноосных пленках при локальном воздействии электрического поля на их поверхность. Показано, что при возрастающем ее воздействии происходит поэтапная трансформация структуры $180^{\circ}$ доменной границы от блоховской к квазиблоховской, а при некотором значения поля и в неелевскую. Выявлено, что в бо́льших полях возможно зарождение $0^{\circ}$ доменной границы с неблоховской структурой, закономерности которого имеют аналогии с процессами образования магнитных неоднородностей на дефектах типа „потенциальная яма“. Определен также вклад парциальных частей неоднородного магнитоэлектрического взаимодействия, обусловленных наличием в них $\operatorname{div} \mathbf{m}$ и $\operatorname{rot} \mathbf{m}$, в рассматриваемые явления.

Работа выполнена при поддержке грантов РФФИ № 16-02-00336-А, № 19-32-50020 мол_нр.

DOI: 10.21883/FTT.2019.06.47688.348

\section{1. Введение}

В последние два десятилетия наблюдается возрастающий интерес к исследованиям магнитоэлектрических материалов, свойства которых можно описать двумя и более взаимодействующими параметрами порядка $[1,2]$. В них был обнаружен ряд необычных и интересных явлений, имеющих перспективы быть примененными в разнообразных технических устройствах спинтроники. В частности предполагается, что эти материалы могут быть использованы в качестве рабочей среды в устройствах магнитной памяти нового поколения (MRAM) [3], в которых запись информации можно осуществить с помощью электрического поля. К такого рода материалам относятся также пленки ферритов гранатов, выделяющиеся среди других тем, что для них имеется развитая технология их получения, причем, с требуемыми свойствами, достигаемая за счет изоморфного замещения ионов в редкоземельных и железных подрешетках [4]. В них впервые был обнаружен гигантский линейный магнитоэлектрический эффект при комнатных температурах [5], а спустя почти два десятилетия новый сильный эффект такого же характера [6]. В последнем случае было установлено, что имеет место смещение доменных границ (ДГ) под действием сильного электрического поля, создаваемого заряженной иглой, поднесенной к поверхности образца. Анализ полученных данных был интерпретирован на основе флексомагнитоэлектрического (ФМЭ) механизма [2,6], который получил в [7] определенное теоретическое обоснование.

Магнитоэлектрический эффект в ферритах-гранатах - явление неординарное, так как кристаллы ферритов-гранатов имеют кубическую симметрию (пространственная группа симметрии $P(m 3 m)$, что запрещает существование магнитоэлектричества в них. Первая интерпретация магнитоэлектрических эффектов, обнаруженных в $\mathrm{Y}_{3} \mathrm{Fe}_{5} \mathrm{O}_{12}$ при низких температуpax [8-10], была связана с понижением симметрии монокристаллов $\mathrm{Y}_{3} \mathrm{Fe}_{5} \mathrm{O}_{12}$ вплоть до моноклинной при понижении температуры. Механизм, объясняющий возникновение электрических свойств ферритов-гранатов за счет поляризации иновалентных ионов $\mathrm{Fe}^{2+}$, был предложен в [11]. В работах [12-14] показано, что наличие магнитной неоднородности высвобождает результирующий дипольный момент, возникающий вследствие полярного механизма, инициирующего возникновение структуры электро-дипольных моментов переходных ионов. Механизм магнитоэлектрических эффектов, обусловленный наличием пространственных неоднородностей вида дефектов, был рассмотрен в $[15,16]$. В рамках механизма, предложенного в [15], предполагается, что существенно неоднородное электрическое поле может привести к индуцированию в области его действия неоднородной анизотропии, которая будет проявляться как дефект. 
В зависимости от направления поля дефект может представлять „потенциальную яму“ или „потенциальный барьер“, в результате чего, ДГ будет притягиваться или отталкиваться от него.

Необходимо отметить, что экспериментальные результаты, полученные в [6], инициировали ряд исследований, среди которых можно выделить [6,7,16-20]. В них изучались различные аспекты проявления ФМЭ эффекта в рассматриваемых в [6] пленках, в частности, в [3] исследовалось зарождение вихрей и антивихрей в электрическом поле, в [7,17] - топология и динамика ДГ, в $[18,19]$ - структура и свойства ДГ с горизонтальными блоховскими линиями, в $[16,20]$ — однородные и неоднородные состояния в (210)-ориентированной пленке ферритов-гранатов.

Известно, что воздействие неоднородного магнитного поля на определенный участок поверхности магнитоодноосной пленки может привести к зарождению в них цилиндрических магнитных доменов треугольной формы [21]. Аналогичное явление было обнаружено и в работе [22], в которой наблюдали зарождение подобных доменов в неоднородном электрическом поле, а также в работе [23], где с помощью сфокусированного лазерного импульсного облучения были индуцированы вертикальные блоховские линии в образце. Поэтому в данной работе будем исходить из того, что сам факт неоднородности поля может повлиять и на зарождение ДГ, и на его трансформацию, и на его смещение [15-17,22].

\section{2. Основные уравнения}

Рассмотрим одноосную ферромагнитную пленку, в которой имеет место неоднородное магнитоэлектрическое взаимодействие (ФМЭ эффект $[2,7,24])$. Будем полагать, что легкая ось анизотропии, а также внешнее электрическое поле $\mathscr{E}$, действующее на пленку, совпадают с нормалью к ее поверхности n. Систему координат выберем таким образом, что бы ось $O z \| \mathbf{n}$, а ось $O y$ совместим с направлением, вдоль которого магнетик неоднороден (рис. 1). В этом случае энергия возможных микромагнитных структур с учетом неоднородного магнитоэлектрического взаимодействия (НМЭВ), приведенная к площади сечения пластины плоскостью $x O z$, запишется в виде [7,17]:

$$
\begin{aligned}
E & =\int_{-\infty}^{\infty}\left\{A\left[\left(\frac{d \varphi}{d y}\right)^{2}+\cos ^{2} \varphi\left(\frac{d \theta}{d y}\right)^{2}\right]\right. \\
& \left.+K_{u}\left(\sin ^{2} \theta \cos ^{2} \varphi+\sin ^{2} \varphi\right)+\varepsilon_{\mathrm{int}}+2 \pi M_{s}^{2} \sin ^{2} \varphi\right\} d y
\end{aligned}
$$

где $\theta, \varphi-$ углы, определяющие единичный вектор намагниченности $\mathbf{m}=\mathbf{M} / M_{s}$ (рис. 1), причем $\mathbf{m}=(\cos \varphi \sin \theta, \sin \varphi, \cos \varphi \cos \theta), A$ - обменный параметр, $K_{u}$ - константа одноосной анизотропии, $M_{s}-$

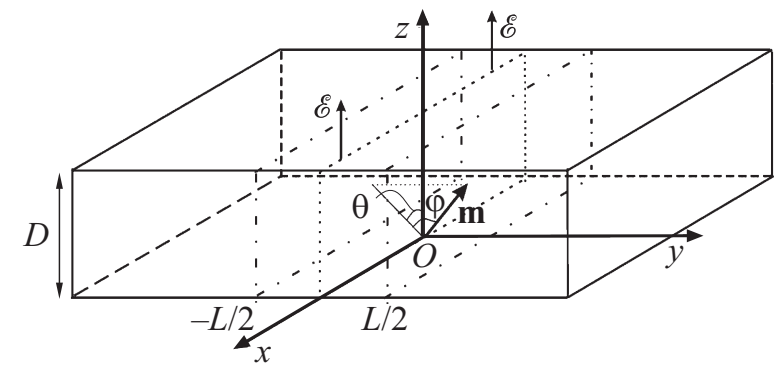

Рис. 1. Геометрия задачи.

намагниченность насыщения, $\varepsilon_{\text {int }}$ - плотность энергии НМЭВ. Считая пластину достаточно толстой, пренебрегаем влиянием размагничивающих полей, обусловленных поверхностными магнитными зарядами, на структуру и свойства магнитных неоднородностей, возможных в данном магнетике. Полагаем, что выражение для $\varepsilon_{\text {int }}$ определяется следующей формулой [25]:

$$
\varepsilon_{\text {int }}=M_{s}^{2} \mathscr{E}\left(b_{1} \mathbf{m} \operatorname{div} \mathbf{m}+b_{2} \mathbf{m} \times \operatorname{rot} \mathbf{m}\right),
$$

которое с учетом выбранного направления $\mathscr{E}$ и системы координат (рис. 1) можно записать в виде

$$
\begin{aligned}
& \varepsilon_{\text {int }}=\mathscr{E} M_{s}^{2} \\
& \times\left[\left(b_{1} \cos ^{2} \varphi+b_{2} \sin ^{2} \varphi\right) \cos \theta \frac{d \varphi}{d y}+b_{2} \sin \theta \sin \varphi \frac{d \theta}{d y}\right],
\end{aligned}
$$

где $b_{1}, b_{2}$ - константы НМЭВ.

Будем считать, что внешнее электрическое поле $\mathscr{E}$, величина которого задается выражением

$$
\mathscr{E}=\mathscr{E}_{0} / \operatorname{ch}(y / L),
$$

действует в ограниченной области пространства; здесь $L$ - определяет размер этой области вдоль оси $O y$, $\mathscr{E}_{0}$ - величину напряженности в центре области действия поля $(y=0)$.

Тогда уравнения Эйлера-Лагранжа, отвечающие минимуму энергии (1) с учетом (3) и записанные в безразмерных величинах, примут вид

$$
\begin{aligned}
& \frac{d}{d \xi}\left(\cos ^{2} \varphi \frac{d \theta}{d \xi}\right)-\sin \theta \cos \theta \cos ^{2} \varphi+\left(\lambda_{1}+\lambda_{2}\right) f(\xi) \\
& \quad \times \sin \theta \cos ^{2} \varphi \frac{d \varphi}{d \xi}+\lambda_{2} \sin \theta \sin \varphi \cos \varphi \frac{d f}{d \xi}=0 \\
& \frac{d^{2} \varphi}{d \xi^{2}}-\sin \varphi \cos \varphi\left[\cos ^{2} \theta-\left(\frac{d \theta}{d \xi}\right)^{2}\right] \\
& +\left(\lambda_{1}+\lambda_{2}\right) f(\xi) \sin \theta \cos ^{2} \varphi \frac{d \theta}{d \xi} \\
& +\left(\lambda_{1} \cos ^{2} \varphi+\lambda_{2} \sin ^{2} \varphi\right) \cos \theta \frac{d f}{d \xi}-Q^{-1} \sin \varphi \cos \varphi=0,
\end{aligned}
$$

где $\quad \lambda_{i}=\mathscr{E}_{0} M_{s}^{2} b_{i} / 2 K_{u} \Delta_{0}-$ приведенное (к характерным величинам $\left.\varepsilon_{i}=2 K_{u} \Delta_{0} / M_{s}^{2} b_{i}, \quad i=1,2\right)$ поле, 
$Q=K_{u} / 2 \pi M_{s}^{2}-$ фактор качества материала, $\xi=y / \Delta_{0}$, $\Delta_{0}=\sqrt{A / K_{u}}, f(\xi)=\mathrm{ch}^{-1}(\xi / l), l=L / \Delta_{0}$.

Полученные уравнения представляют собой существенно нелинейные дифференциальные уравнения II порядка с непостоянными коэффициентами; они описывают структуру и свойства магнитных неоднородностей, возможных в рассматриваемом магнетике. Очевидно совместное решение уравнений системы (5) аналитически не представляется возможным из-за непреодолимых трудностей, возникающих при анализе таких уравнений, содержащих наряду со вторыми $\left(\theta^{\prime \prime}, \varphi^{\prime \prime}\right)$ и первые производные $\left(\theta^{\prime}, \varphi^{\prime}\right)$, в том числе и члены содержащие $\left(\theta^{\prime}\right)^{2}$, а также функции, зависящие от координат в явном виде $\left(f(y), f^{\prime}(y)\right)$. Поэтому в дальнейшем искать решения этих уравнений будем путем их численного интегрирования.

\section{3. Трансформация структуры $180^{\circ}$ ДГ}

В основе численного исследования уравнений (5) был положен метод множественной стрельбы с применением итерационной процедуры по Ньютону [26], апробированный в [27]. Из результатов численной реализации задачи для случая НМЭВ с $b_{1}=b_{2}$, следует, что при действии электрического поля на образец, происходит изменение топологии $180^{\circ}$ ДГ: она из ДГ блоховского типа $(\varphi=0)$ преобразуется в ДГ с некруговой траекторией вектора намагниченности $(\varphi=\varphi(y))[28,29]$, т.е. с выходом вектора $\mathbf{m}$ из плоскости вращения магнитных моментов (рис. 2). Последнее означает, что ДГ благодаря ФМЭ механизму [2,7] становится заряженной. При этом величина дифференциальной поляризации ДГ $p$, определяемая по формуле

$$
p=-\frac{\partial \varepsilon_{\text {int }}}{\partial \varepsilon},
$$

будет уже отличной от нуля (в данном случае $p>0$ ) и стенка будет притягиваться к источнику поля. Величина $p$ является четной ограниченной функцией от $y$ (рис. 3), достигающей максимального значения $p_{m}$ в центре стенки. Здесь $p=v p_{0}$, где $p_{0}=\left(M_{s}^{2} b_{i} / \Delta_{0}\right.$ и $v-$ соответственно, характерная и приведенная величины поляризации. Аналогичная зависимость от $у$ имеет место и для $\varphi=\varphi(y)$ (рис. 2,a). Отсюда следует, что чем больше величина поля $\varepsilon_{0}$, тем больше максимальный угол выхода $\mathbf{m}$ из плоскости стенки $\left(\varphi_{m}\right)$, а вместе с ним и величина $p_{m}$.

Согласно расчетам при дальнейшем возрастании $\mathscr{E}_{0}$ максимальный угол выхода $\varphi_{m}=\varphi(0)$ увеличивается и при определенном значении поля $\mathscr{E}_{0}=\lambda_{i} \mathscr{E}_{i}=0.4 \mathscr{E}_{1}$ (т. к. $b_{1}=b_{2}$, то $\mathscr{E}_{1}=\mathscr{E}_{2}, \lambda_{1}=\lambda_{2}=0.4$ ), становится равным $\pi / 2$, то есть в ней появляется неелевский участок закона поворота $\mathbf{m}$ в переходном слое (вблизи $y=0$ ). Последний затем расширяется и при достижении полем величины $\mathscr{E}_{0 c}=0.488 \mathscr{E}_{1} 180^{\circ}$ ДГ полностью становится неелевской (рис. 4). Полученный результат согласуется
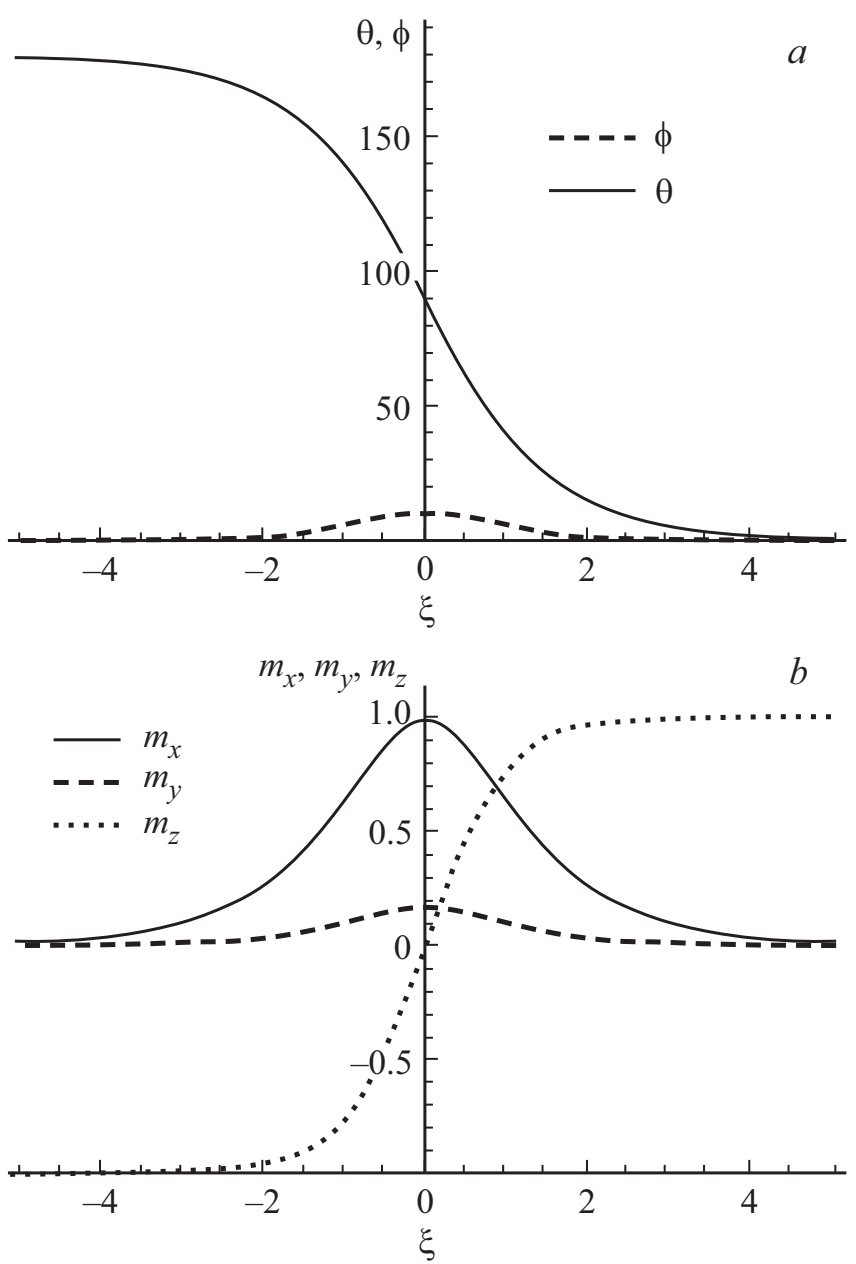

Рис. 2. Графики, иллюстрирующие распределение намагниченности $\mathbf{m}$ в $180^{\circ}$ ДГ, представленные через зависимости ее угловых переменных $\theta, \varphi(a)$ и через ее компоненты $m_{x}, m_{y}$, $m_{z}(b)$ от $\xi$ при следующих значениях параметров: $\lambda=0.1$ $\left(b_{1}=b_{2}\right), Q=3, l=5$.

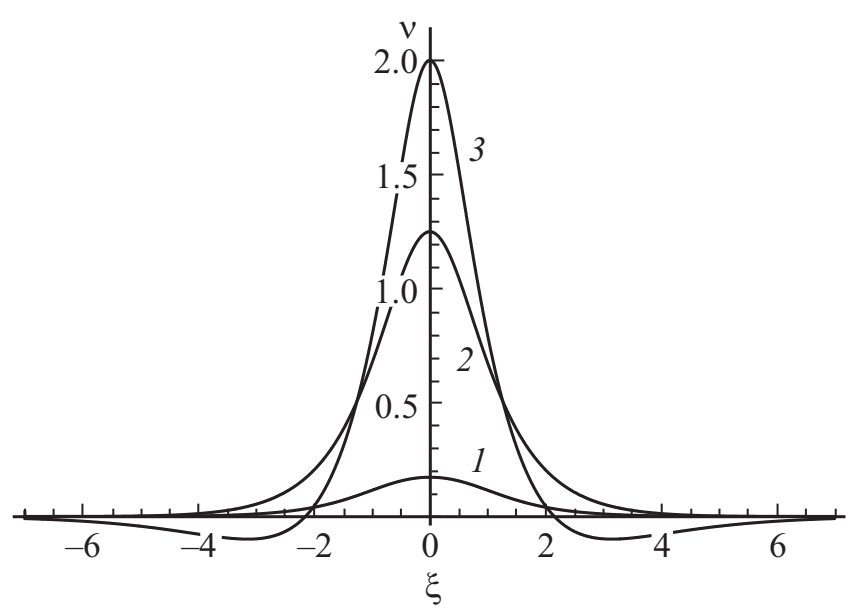

Рис. 3. График зависимости величины $v$, определяющей дифференциальную поляризацию $180^{\circ}$ ДГ, от $\xi$. Линия 1 соответствует $\lambda=0.1,2-\lambda=0.488,3-\lambda=5$; остальные параметры те же, что и на рис. 2. 
с расчетами $[17,25]$, в которых также утверждается, что структура $180^{\circ}$ ДГ в однородном электрическом поле, превышающем некоторое критическое значение, становится неелевской. Следует отметить, что в этих работах анализ трансформации структуры стенки не рассматривался.

Исследования показывают (рис. 5), что критическое поле перехода в неелевскую стенку $\mathscr{E}_{0 c}=\lambda_{c} \mathscr{E}_{1}$ существенным образом зависит от размера области неоднородности электрического поля $L$ : с уменьшением $L$ величина $\mathscr{E}_{0 c}$ возрастает, а при $L \rightarrow 0$, значение критического поля $\mathscr{E}_{0 c}$ становится неограниченным $\left(\mathscr{E}_{0 c} \rightarrow \infty\right)$, с другой стороны, при возрастании $L$ величина $\mathscr{E}_{0 c}$ уменьшается и при $L \rightarrow \infty$ достигает некоторого предельного значения, совпадающего со значением $\mathscr{E} 0 c$ в случае действия однородного поля $\mathscr{E}$ на доменную стенку. Такая зависимость вполне объяснима тем вкладом, которое вносит НМЭВ в общую энергию (1). В частности,

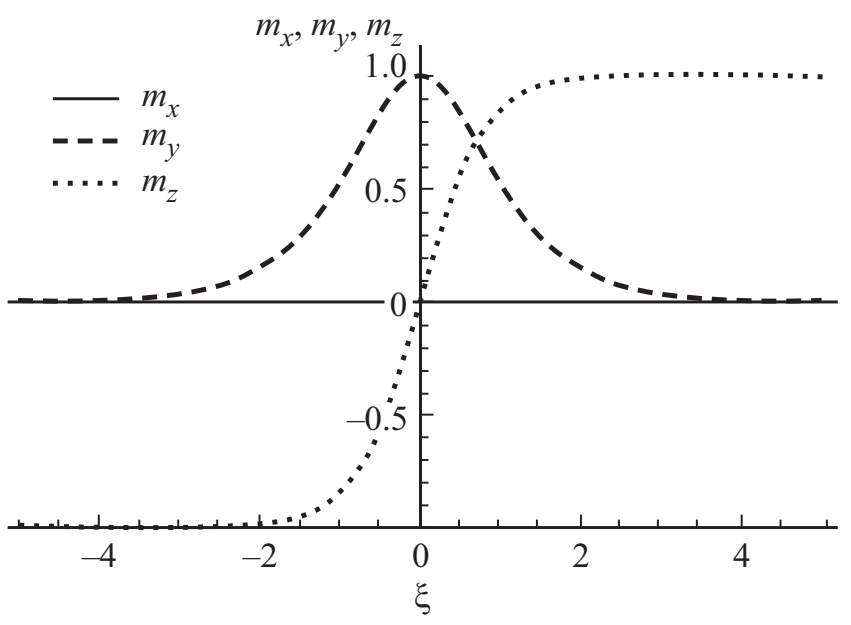

Рис. 4. Графики зависимости компонент $m_{x}, m_{y}, m_{z}$ от $\xi$ при $\lambda=0.488\left(b_{1}=b_{2}\right)$; остальные параметры те же, что и на рис. 2 .

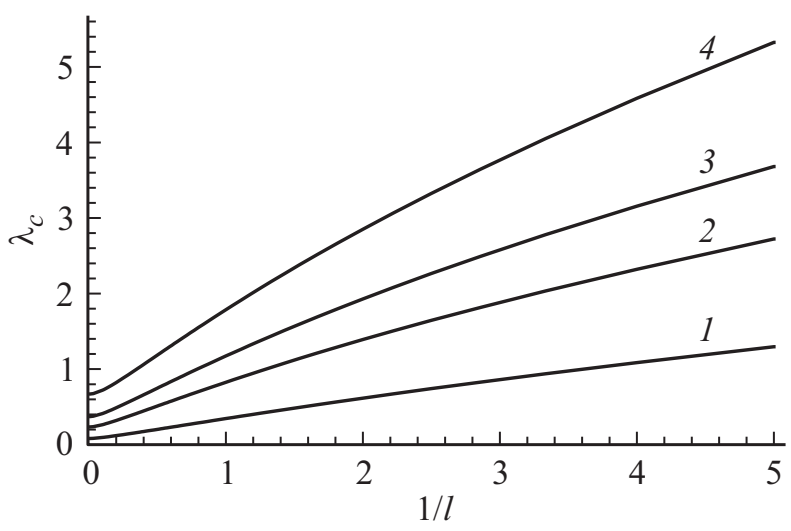

Рис. 5. Графики зависимости величины $\lambda_{c}$, определяющей критические поля перехода $180^{\circ}$ ДГ из квазиблоховской структуры в неелевскую от величины обратной $l$. Линия 1 соответствует $Q=15$, линия $2-Q=5$, линия $3-Q=3$, линия $4-Q=1.5$.

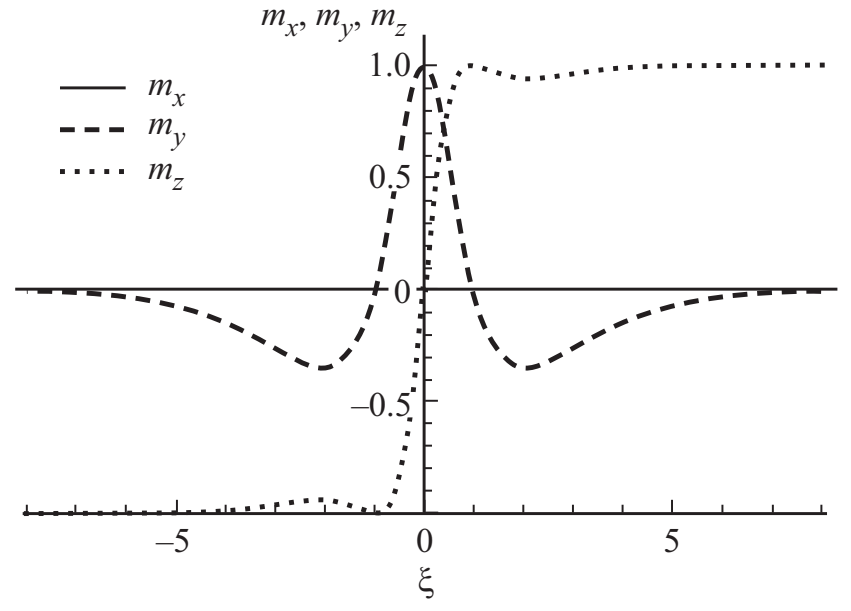

Рис. 6. График зависимости компонент $m_{x}, m_{y}, m_{z}$ от $\xi$ при $\lambda=6$. Остальные параметры те же, что и на рис. 2.

энергия НМЭВ, определяемая выражением

$$
E_{\mathrm{int}}=\int_{-\infty}^{\infty} \varepsilon_{\mathrm{int}} d y,
$$

пропорциональна размеру области действия неоднородного электрического поля $L$, а также величине $\mathscr{E} 0$. Отсюда следует, что $E_{\text {int }}=c L \mathscr{E}_{0 c}$, где $c-$ некоторая константа. Таким образом, можно утверждать, что чем меньше размер области действия поля $L$, тем большей величины необходимо приложить поле, чтобы $180^{\circ}$ ДГ стала неелевской и наоборот. В силу того, что при значении поля $\mathscr{E}=\mathscr{E}_{0 c}$ структура $180^{\circ}$ ДГ будет всегда неелевской, то константа $c$ практически не будет зависеть от $L$.

При дальнейшем увеличении величины $\mathscr{E}_{0}$ структура $180^{\circ}$ ДГ претерпевает ряд трансформаций, связанных с возникновением дополнительных экстремумов на графиках зависимостей компонент вектора m от координаты $y$ (рис. 6). При этом стенка остается неелевской, а величина $p_{m}$ непрерывно растет. Соответственно, интегральная величина поляризации $P=N p_{0}$, определяемая по формуле

$$
P=\int_{-\infty}^{\infty} p(y) d y,
$$

также будет увеличиваться.

До сих пор предполагалось, что вклады параметров $b_{1}$ и $b_{2}$, которые определяют интенсивности соответствующих частей НМЭВ в ФМЭ эффект, одинаков $\left(b_{1}=b_{2}\right)$ [24]. В тоже время, выражение для плотности энергии НМЭВ, представленное в [25] в виде (2), предполагает, что эти вклады возможно отличаются. Поэтому необходимо выяснить в рамках данной задачи степень влияния каждой части НМЭВ на структуру и поляризацию ДГ. 
Из расчетов следует, что в малых полях эти вклады носят аддитивный характер [7], но различаются по характеру их воздействия на ДГ. В частности, вклад, обусловленный наличием в $\varepsilon_{\text {int }}$ слагаемого, содержащего $\operatorname{rot} \mathbf{m}$ (второй тип), приводит к более существенным изменениям структуры (увеличение $\varphi_{m}$ и ширины угловой зависимости $\varphi=\varphi(y)$ происходит сильнее рис. 7), чем вклад, связанный с $\operatorname{div} \mathbf{m}$ (первый тип). Однако их вклады в дифференциальную поляризацию $p$ отличаются не только количественно, но и качественно. Так поляризация $p$, обусловленная парциальным вкладом НМЭВ второго типа, имеет распределение по координате $y$ (рис. 8), коррелирующее с угловой зависимостью $\varphi=\varphi(y)$ (рис. 7). В то же время распределение поляризации, обусловленное первым типом НМЭВ, описывается также четной ограниченной функцией, но с двумя

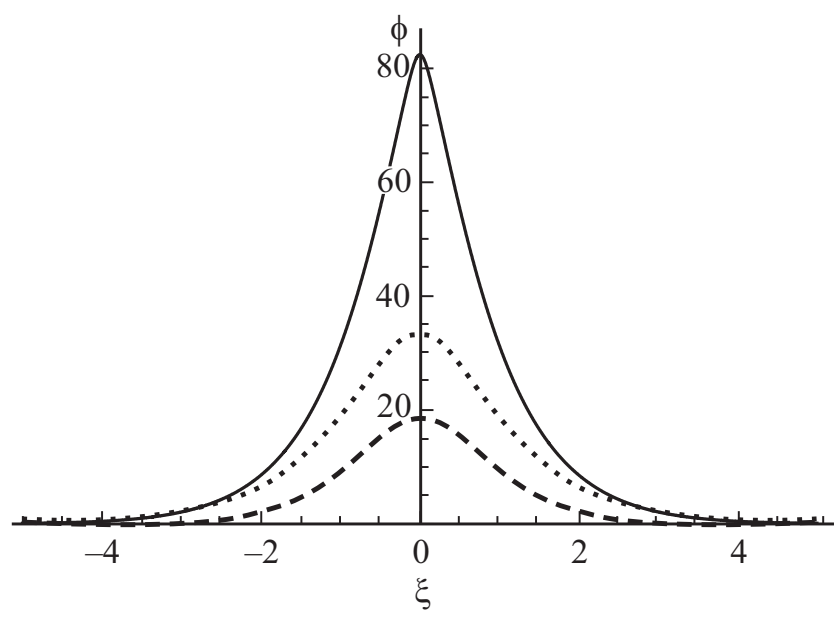

Рис. 7. Графики зависимости $\varphi$ от $\xi$ для $180^{\circ}$ ДГ при $\lambda=0.485, Q=3, l=5$. Здесь штриховая линия соответствует случаю, когда в НМЭВ - $b_{1} \neq 0, b_{2}=0$, пунктирная $b_{1}=0, b_{2} \neq 0$, сплошная $-b_{1}=b_{2} \neq 0$ (совместный вклад обоих типов НМЭВ).

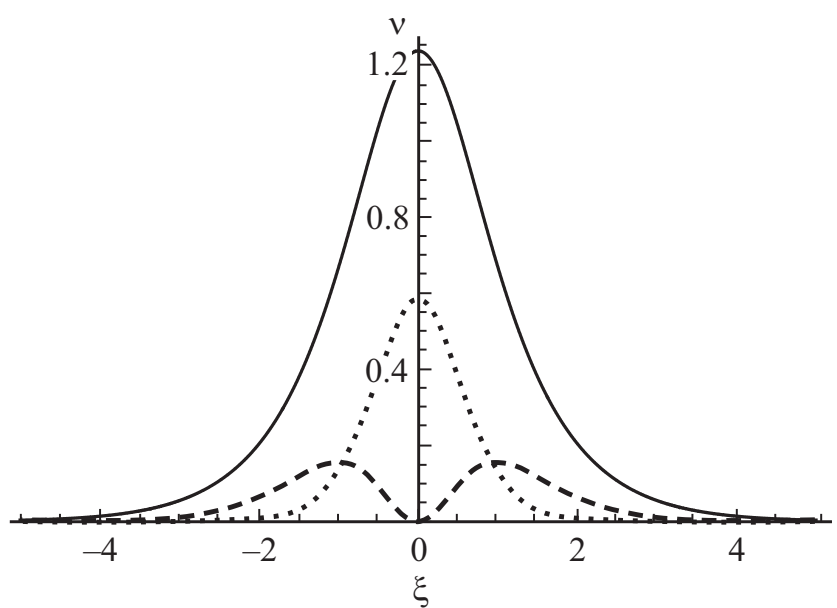

Рис. 8. График зависимости параметра $v$ для $180^{\circ}$ ДГ от $\xi$. Значения параметров материала и обозначения кривых те же, что на рис. 7.

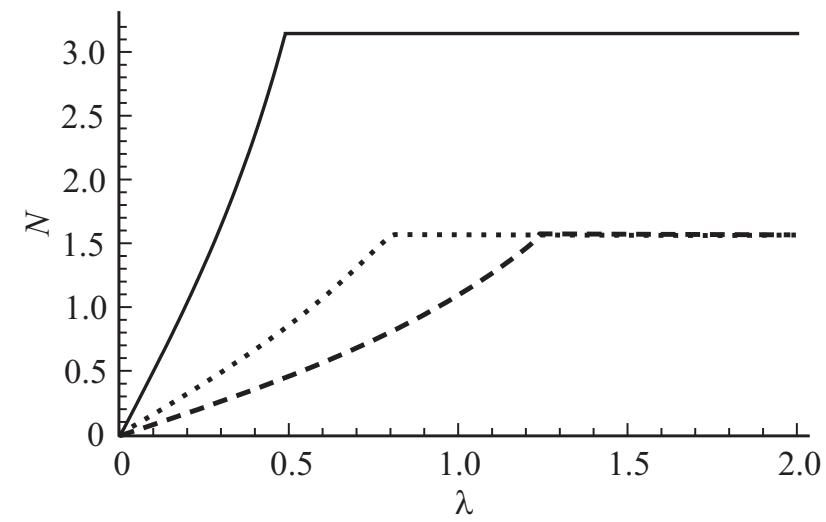

Рис. 9. Графики зависимости параметра $N$ для $180^{\circ}$ ДГ от приведенного поля $\lambda$ при $Q=3, l=5$. Сплошная линия соответствует случаю учета НМЭВ с $b_{1}=b_{2} \neq 0$, штриховая $b_{1} \neq 0, b_{2}=0$, пунктирная $-b_{1}=0, b_{2} \neq 0$.

пиками, симметрично расположенными относительно оси ординат и с $p(0)=0$. Таким образом, в первом случае при включении поля в ДГ может возникнуть двойной электрический слой. $\mathrm{B}$ случае же $b_{1}=b_{2}$ их совместное действие также приводит к гауссовскому распределению величины $v$ с максимальным значением поляризации в центре стенки. Влияние первого типа НМЭВ на результирующую поляризацию приводит лишь к некоторому уширению функции $p=p(y)$ в местах, где первой тип НМЭВ дает максимальный вклад в нее. Можно отметить, что в бо́льших полях их вклады уже не являются аддитивными (это особенно заметно при анализе величины $p_{m}$ на рис. 8). В данном случае они значительно усиливают друг друга, что связано с нелинейным характером уравнений (5). При этом можно отметить, что при значении поля, равным $\mathscr{E}_{0 c}$, на графиках зависимости $p_{m}$ от $\mathscr{E}_{0}$ для обоих вкладов имеется излом: характер зависимостей меняется от крутого подъема при малых $\mathscr{E}_{0}<\mathscr{E}_{0 c}$, до более пологого возрастания при бо́льших $\mathscr{E}_{0}>\mathscr{E}_{0 c}$. Аналогично ведет и величина интегральной поляризации $P$ (рис. 9), что связано с перестройкой структуры $180^{\circ}$ ДГ: она трансформируется от квазиблоховского типа к неелевскому.

\section{0-градусные ДГ}

Анализ уравнений Эйлера-Лагранжа (5) показывает, что в неоднородном электрическом поле вида (4) возможны также решения, соответствующие 0-градусным ДГ $\left(0^{\circ}\right.$ ДГ). Они представляют собой магнитную неоднородность, разделяющую два домена с одинаковым направлением намагниченности $\mathbf{m}_{0}$ в них и имеющую гауссовский характер зависимости $\theta=\theta(y)$ [27,30]]. $0^{\circ}$ ДГ имеют важное значение в процессах спиновой переориентации магнетика от одного направления к другому, в которых они играют роль зародышей перемагничивания [30,31]. Кроме того, такие неоднородности 
могут образоваться в магнетиках на дефектах типа „потенциальная яма“ [27]. В данной ситуации возникновение $0^{\circ}$ ДГ в одноосных ферромагнетиках с НМЭВ в неоднородном электрическом поле является вполне закономерным явлением, так как действие такого рода поля на магнитные моменты ограничено в пространстве и проявляет себя как дефект, индуцированный электрическим полем. Конечно механизм его воздействия на магнетик может быть двояким и привести еще к неоднородному смещению однотипных ионов и в конечном счете к индуцированию дополнительной анизотропии, которая будет также пространственно неоднородной $[15,16]$. Однако в данном случае ограничимся рассмотрением только первого механизма.

Таким образом зарождение $0^{\circ}$ ДГ в неоднородном электрическом поле, как и в случае зарождения $0^{\circ}$ ДГ на дефектах типа „потенциальная яма“, изученным в [27], носит пороговый характер, так как $0^{\circ}$ ДГ появляются только в полях $\mathscr{E} 0$, превышающих некоторое критическое значение $\mathscr{E}_{0 n}$ (в частности, при $\mathscr{E}_{0}>\mathscr{E}_{0 n}=0.719 \mathscr{E}_{1}$ для случая $L=10 \Delta_{0}, Q=3, b_{1}=b_{2}$, рис. 10). Справедливости ради надо отметить, что $0^{\circ}$ ДГ в рассматриваемым магнетике может зародиться и в однородном поле. Однако его энергия при этом будет положительной и следовательно, такая $0^{\circ}$ ДГ не будет устойчивой.

Как видно из рис. 10, в момент зарождения $0^{\circ}$ ДГ имеет неблоховскую структуру, в которой зависимость $\varphi=\varphi(y)$ является функцией нечетной. Последнее означает, что вполне достаточно исследовать область $y>0$. В этом случае, как следует из рис. 10, в момент своего зарождения (при $\left.\mathscr{E}_{0}=\mathscr{E}_{0 n}\right)$ функция $\varphi(y)$ имеет в области $y>0$ при $y=y_{1}$ лишь один максимум $\varphi_{m}=\varphi\left(y_{1}\right)$, который является немалой величиной: $\varphi_{m} \sim 70^{\circ}$. Однако при возрастании $\mathscr{E}_{0}$ на графике зависимости $\varphi=\varphi(y)$ (рис. 10, кривая $2^{\prime}$ ) появляется еще один минимум $\left(y=y_{2}\right)$, который расположен в области $\varphi(y)<0$, причем его координата находится правее координаты максимума функции $\varphi(y)\left(y_{1}<y_{2}\right)$. Очевидно, при $y>y_{2}$ на графике зависимости имеется точка перегиба $y_{p}$, которая, согласно [32], определяет ширину $\Delta$ угловой зависимости $\varphi=\varphi(y)$.

При возрастании величины поля $\mathscr{E}_{0}$ координата $y_{2}$ точки минимума функции $\varphi(y)$, а вместе с ним и точки перегиба $y_{p}$ смещаются в сторону меньших значений $y$. При этом глубина минимума $\left|\varphi\left(y_{2}\right)\right|$ растет при одновременном уменьшении высоты максимума $\varphi\left(y_{1}\right)$, а ширина $\Delta$ также уменьшается. При больших значениях $\mathscr{E}_{0}$ (в частности, для значений $L=5 \Delta_{0}, Q=3$, при $\left.\mathscr{E}_{0}>9.3 \mathscr{E}_{1}\right)$ происходит возникновение допольнительного максимума и одного минимума на графике зависимости $\theta=\theta(y)$. Такая существенная трансформация структуры $0^{\circ}$ ДГ, которая происходит при увеличении напряженности $\mathscr{E}_{0}$, приводит к уменьшению поляризации ДГ, как величины $p(0)$, так и ее интегральной величины $P$.

Значение поля зарождения $\mathscr{E}_{0 n}=\lambda_{n} \mathscr{E}_{1}$ существенно зависит от размера $L$ области действия неоднородного

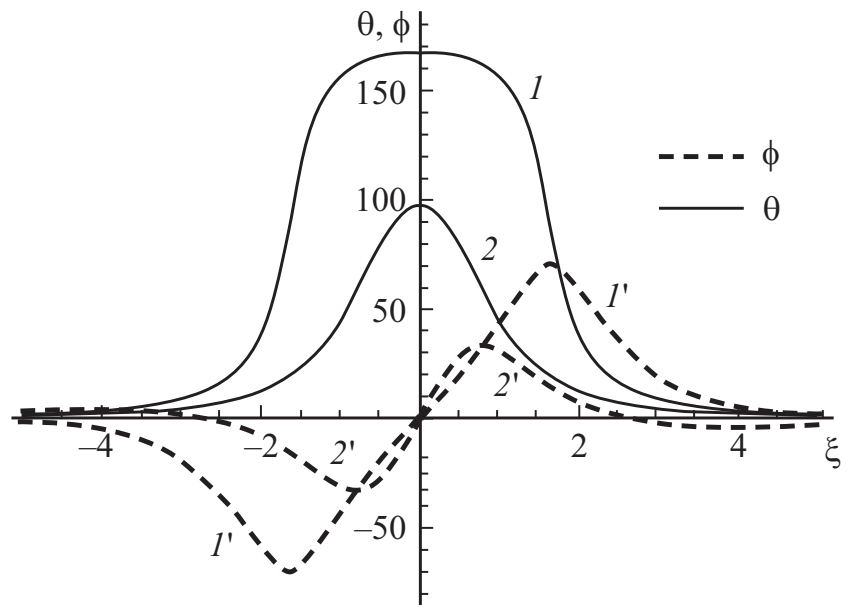

Рис. 10. Графики, иллюстрирующие распределение намагниченности $\mathbf{m}$ в $0^{\circ}$ ДГ, выраженные через зависимости $\theta, \varphi$ от $\xi$ при $\lambda=0.719$ (линии 1 и $l^{\prime}$ ) и $\lambda=2$ (линии 2 и $2^{\prime}$ ). Здесь $b_{1}=b_{2}, Q=3, l=10$.

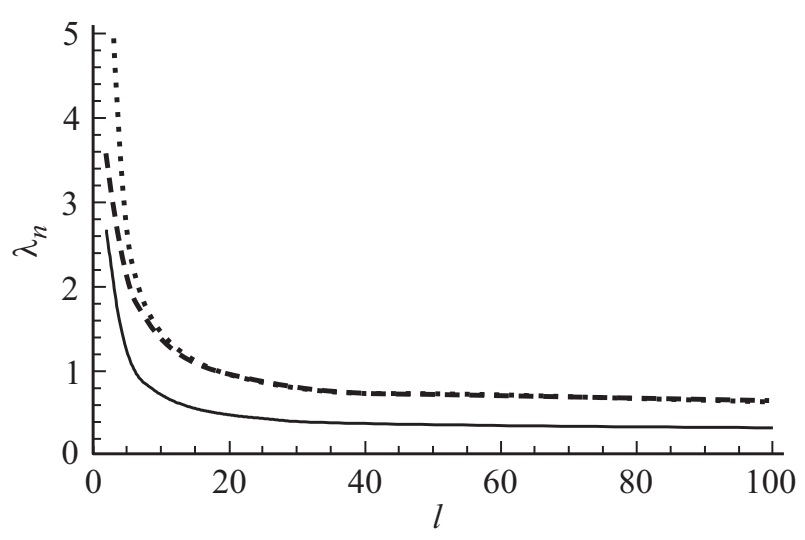

Рис. 11. Графики зависимости параметра $\lambda_{n}$ от величины $l$ для $0^{\circ}$ ДГ при $Q=3$. Штриховая линия соответствует случаю учета вклада НМЭВ первого типа, пунктирная — второго типа, сплошная - случаю учета совместного вклада НМЭВ.

поля (рис. 11): чем больше величина $L$, тем при меньших критических полях зарождаются $0^{\circ}$ ДГ и наоборот. Следует отметить, что аналогичная зависимость имеет место и при рассмотрении в одноосных пленках условий возникновения $0^{\circ}$ ДГ на дефектах типа „потенциальная яма“ $[27,30,33]$. С другой стороны с возрастанием размера $L$ амплитуда $\theta_{m}$ и поляризация $p_{m}$ уменьшаются. Кроме того на графике зависимости дифференциальной поляризации $p$ от координаты (рис. 12) в момент зарождения $0^{\circ}$ ДГ имеются два максимума $p_{m}$, симметрично расположенных относительно начала координат, и один относительный минимум (между ними в точке $y=0$ ), которые с возрастанием величины поля $\mathscr{E}_{0}$ одновременно уменьшаются. При этом при возрастании $L$ значение $p(0)$ также уменьшается, как и интегральная величина поляризации $P$. 


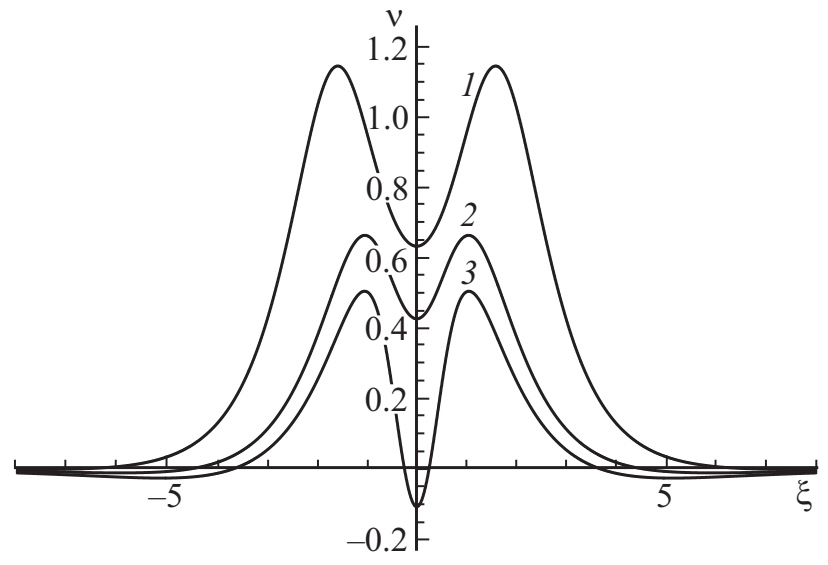

Pис. 12. Графики зависимости величины $v$ для $0^{\circ}$ ДГ от $\xi$ при $Q=3, l=5, b_{1}=b_{2}$. Линия 1 соответствует $\lambda=0.719,2-$ $\lambda=1.5,3-\lambda=2.5$.

Необходимо отметить, что приведенные выше явления зарождения $0^{\circ}$ ДГ и дальнейшей его трансформации в неоднородном электрическом поле изучались для случая $b_{1}=b_{2}$. Однако расчеты показывают, что влияние парциальных вкладов НМЭВ, в структуру $0^{\circ}$ ДГ существенно отличаются от той картины, которая имела место для $180^{\circ}$ ДГ. В частности, поле зарождения $0^{\circ}$ ДГ в исследуемом магнетике, найденное при учете лишь вклада НМЭВ первого типа $\left(b_{1} \neq 0, b_{2}=0\right)$, ощутимо меньше $\left(\mathscr{E}_{0 n}=2.173 \mathscr{E}_{1}\right.$, при $\left.L=5 \Delta_{0}, Q=3\right)$, чем поле зарождения - при учете вклада второго типа $\left(\mathscr{E}_{0 n}=2.737 \mathscr{E}_{2}\right.$, $b_{1}=0, \quad b_{2} \neq 0, L$ и $Q-$ те же значения). Более того характеристики $0^{\circ}$ ДГ при учете различных типов НМЭВ, в момент зарождения также значительно отличаются: в первом случае $\theta_{m} \sim 165^{\circ}, \varphi_{m} \sim 56^{\circ}, \Delta=5 \Delta_{0}$ (эффективный размер $0^{\circ}$ ДГ согласно [32]), а во втором случае $\theta_{m} \sim 175^{\circ}, \varphi_{m} \sim 62^{\circ}, \Delta=2.6 \Delta_{0}$. С возрастанием значения электрического поля $\mathscr{E}_{0}$ величины $\theta_{m}$ и $\varphi_{m}$ в обоих случаях уменьшаются, однако данная тенденция происходит для первого случая более замедлено, чем для второго случая. Кроме того, обнаруженное ранее возникновение еще одного минимума на графиках зависимости при $y>0$ с возрастанием $\mathscr{E}_{0}$ имеет место только в случае учета вклада НМЭВ первого типа, причем значение этого минимума $\left(\left|\varphi\left(y_{2}\right)\right|\right)$ увеличивается с возрастанием $\mathscr{E}_{0}$. Таким образом, можно утверждать, что часть НМЭВ, обусловленного наличием в нем $\operatorname{div} \mathbf{m}$, приводит к более существенной трансформации структуры $0^{\circ}$ ДГ, чем та часть НМЭВ, в выражении которого содержится rot $\mathbf{m}$. Отсюда вытекает, что основной вклад в величину индуцированной поляризации $0^{\circ}$ ДГ вносит первый тип НМЭВ.

\section{5. Обсуждение результатов}

Анализ полученных данных показывает, что наложение электрического поля, направленного нормально к поверхности пленки и действующего в ограниченной ее области, приводит к трансформации распределения намагниченности в $180^{\circ}$ ДГ: ее структура (первоначально блоховского типа) преобразуется в квазиблоховскую с выходом вектора намагниченности $\mathbf{m}$ из плоскости стенки [20,29]. При этом благодаря ФМЭ механизму ДГ становится заряженной, величина поляризации которой описывается четной, ограниченной функцией гауссовского типа. Дальнейшее увеличение поля приводит к возрастанию одновременно интегральной поляризации $P$ и максимального угла $\varphi_{m}$. Последний все время увеличивается вплоть до значения $\varphi_{m}=\pi / 2$, что означает возникновение в структуре ДГ неелевского участка, который затем разрастается. Наконец, при достижении полем некоторого критического значения $\mathscr{E}_{0 c} \quad 180^{\circ}$ ДГ становится полностью неелевской. При другом значении поля $\mathscr{E}_{0}=\mathscr{E}_{0 n}$, немного бо́льшим по величине, чем $\mathscr{E}_{0 c}$, зарождается магнитная неоднородность типа $0^{\circ}$ ДГ, которая уже в момент ее образования является неблоховской $(\operatorname{div} \mathbf{m} \neq 0)$. Она, соответственно, имеет определенную поляризацию, интегральная величина которой с возрастанием $\mathscr{E}_{0}$ также увеличивается. Согласно расчетам, поле зарождения $0^{\circ}$ ДГ $\mathscr{E}_{0 n}$ существенно зависит от ширины области действия поля $L$ : чем больше $L$, тем меньше $\mathscr{E}_{0 n}$ и при $L \rightarrow \infty$ поле $\mathscr{E}_{0 n}$ асимптотически быстро стремится к величине $\mathscr{E}_{0 n}=0.3 \mathscr{E}_{1}$. Таким образом, поля $\mathscr{E}_{0 c}$ и $\mathscr{E}_{0 n}$ являются характерными величинами, при достижении которых качественно меняются структура и свойства магнитных неоднородностей.

Следует отметить, что общая картина поведения микромагнитной структуры в неоднородном электрическом поле согласуется с экспериментальными данными, однако детальное сравнение результатов, полученных в данной работе с данными эксперимента $[6,15]$ не представляется возможным в связи с существенными различиями в геометрии пленок: в работе [6] изучались доменные границы в (210) ориентированных пленках ферритов-гранатов, в которых значительную роль играет ромбическая и „скошенная ромбическая“ анизотропии [20,34], а здесь анализ магнитных неоднородностей рассмотрен в одноосном ферромагнетике.

Закономерности зарождения $0^{\circ}$ ДГ в магнитоодноосной пленке в неоднородном электрическом поле, полученные в данной работе, во многом идентичны процессам образования $0^{\circ}$ ДГ на дефектах типа „потенциальная яма“ [33]. Еще одним фактором, подтверждающим сходство этих явлений является зависимость энергии $0^{\circ}$ ДГ от величины поля $\mathscr{E}_{0}$ : при определенном его значении энергия $0^{\circ}$ ДГ становится отрицательной (например, для $Q=3, L=10 \Delta_{0}$ при $\mathscr{E}_{0}>5.4 \mathscr{E}_{1}$, (рис. 13)) и, следовательно, ее образование становится энергетически более выгодным, чем однородное состояние магнетика (как и в случае с $0^{\circ}$ ДГ, зарождающимися на соответствуюших дефектах $[27,33])$. Таким образом действие неоднородного электрического поля на магнетик, в котором имеет место НМЭВ, аналогично по вызываемым последствиям наличию в образце дефекта типа „потенциальная яма“. 


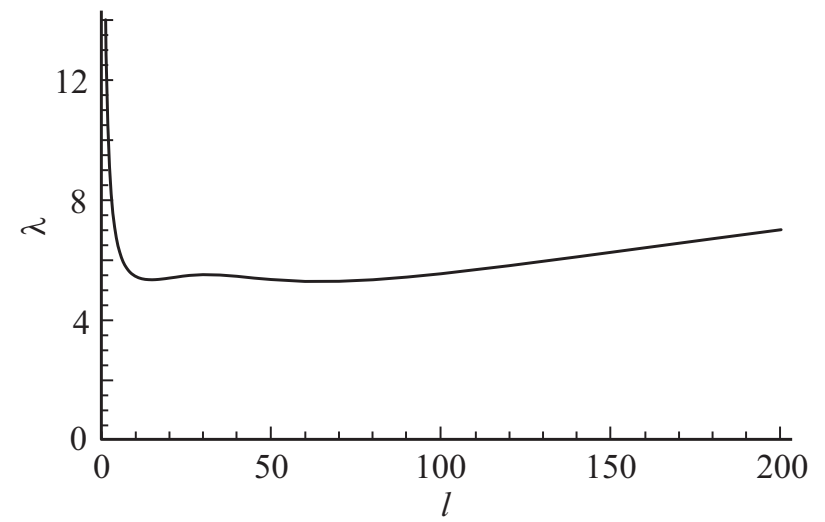

Рис. 13. График зависимости параметра $\lambda$ от $l$, представленная в виде линии $\lambda(l)$, на которой энергия $0^{\circ}$ ДГ обращается в ноль; в области, расположенной выше этой линии энергия $0^{\circ}$ ДГ отрицательна, а ниже - положительна.

Однако есть и отличия, которые заключаются в следующем. Во-первых, ФМЭ эффект всегда приводит к трансформации существующих или к образованию новых магнитных неоднородностей таким образом, чтобы они соответствовали неблоховской структуре, в результате чего на ДГ возникает поляризация. Во-вторых, $0^{\circ}$ ДГ при наличии в магнетиках НМЭВ может возникать в них и в однородном электрическом поле, хотя ее энергия является положительной.

Следует отметить, что в работе исследовалось также парциальные вклады НМЭВ, обусловленные наличием в (2) соответствующих слагаемых (div m и $\operatorname{rot} \mathbf{m})$, в структуру магнитных образований, возникающих в неоднородном поле. Выяснилось, что на $180^{\circ}$ ДГ более сильное влияние оказывает часть НМЭВ второго типа, а на $0^{\circ}$ ДГ - часть НМЭВ первого типа. Такая избирательность вкладов разного типа возможно объясняется тем, что НМЭВ второго типа, легче изменить структуру $180^{\circ}$ ДГ, в которой уже имеется ,завихрение“ магнитных моментов (точнее замыкание магнитного потока в ДГ), чем структуру $0^{\circ}$ ДГ, не обладающей такой особенностью.

В заключение авторы выражают признательность профессору физического факультета МГУ А.П. Пятакову за проявленный интерес к нашей работе и предоставленные экспериментальные материалы.

\section{Список литературы}

[1] G. Catalan, J. Seidel, R. Ramesh, J.F. Scott. Rev. Mod. Phys. 84, 119 (2012).

[2] А.П. Пятаков, А.К. Звездин. УФН 182, 593 (2012).

[3] G.A. Meshkov, A.P. Pyatakov, A.D. Belanovsky, K.A. Zvezdin, A.S. Logginov. J. Magn. Soc. Jpn. 36, 46 (2012).

[4] В.В. Рандошкин, А.Я. Червоненкис. Прикладная магнитооптика. Энергоатомиздат, М. (1990). 320 с.

[5] Б.Б. Кричевцов, В.В. Павлов, Р.В. Писарев. Письма ЖЭТФ 49, 466 (1989).
[6] А.С. Логгинов, Г.А. Мешков, А.В. Николаев, А.П. Пятаков. Письма ЖЭТФ 86, 2 (2007).

[7] Р.М. Вахитов, А.Т. Харисов, Ю.Е. Николаев. ДАН. Физика 455, 150 (2014).

[8] T. O’Dell. Philosoph. Mag. 16, 487 (1967).

[9] G. Velleaud, B. Sangare, M. Mercier, G. Aubert. Solid State Commun. 52, 71 (1984).

[10] H. Ogawa, E. Kita, Y. Mochida, K. Kohn, S. Kimura, A. Tasaki, K. Siratori. J. Phys. Soc. Jpn 56, 452 (1987).

[11] Y. Kohara, Y. Yamasaki, Y. Onose, Y. Tokura, Phys. Rev. B 82, 104419 (2010).

[12] A.I. Popov, Z.V. Gareeva, A.K. Zvezdin. Phys. Rev. B 92, 144420 (2015).

[13] A.I. Popov, K.A. Zvezdin, Z.V. Gareeva, F.A. Mazhitova, R.M. Vakhitov, A.R. Yumaguzin, A.K. Zvezdin. J. Phys.: Condens. Matter. 28, 456004 (2016).

[14] A.I. Popov, Z.V. Gareeva, F.A. Mazhitova, R.A. Doroshenko. JMMM 461, 128, (2018).

[15] А.Ф. Кабыченков, Ф.В. Лисовский, Е.Г. Мансветова. Письма ЖЭТФ 97, 304 (2013).

[16] Г.В. Арзамасцева, А.М. Балбашов, Ф.В. Лисовский, Е.Г. Мансветова, А.Г. Темирязев, М.П. Темирязева. ЖЭТФ 147, 793 (2015).

[17] М.А. Шамсутдинов, А.Т. Харисов, Ю.Е. Николаев. ФММ 111, 472 (2011).

[18] М.А. Борич, А.П. Танкеев, В.В. Смагин. ФТТ 58, 63 (2016).

[19] М.А. Борич, А.П. Танкеев, В.В. Смагин. ФТТ 58, 1329 (2016).

[20] Р.М. Вахитов, Р.Р. Исхакова, А.Р. Юмагузин. ФТТ 60, 923 (2018).

[21] А.П. Иванов, А.С. Логгинов, Г.А. Непокойчицкий, И.И. Никитин. ЖЭТФ 84,1006 (1984).

[22] Д.П. Куликова, А.П. Пятаков, Е.П. Николаева, А.С. Сергеев, Т.Б. Косых, З.А. Пятакова, А.В. Николаев. Письма в ЖЭТФ 104, 196 (2016).

[23] А.С. Логгинов, А.В. Николаев, Е.П. Николаева, В.Н. Онищук. ЖЭТФ 117, 571 (2000).

[24] В.Г. Барьяхтар, В.А. Львов, Д.А. Яблонский. Письма ЖЭТФ 37, 565 (1983).

[25] I.E. Dzyaloshinskii. Europhys. Lett. (EPL) 83, 67001 (2008).

[26] Дж. Холл, Дж. Уатт. Современные численные методы решения обыкновенных дифференциальных уравнений. Мир, М. (1979). 372 c.

[27] Р.М. Вахитов, Т.Б. Шапаева, Р.В. Солонецкий, А.Р. Юмагузин. ФММ 118, 571 (2017).

[28] A. Hubert, R. Schäfer. Magnetic domains. Springer-Verlag, Berlin (2009). 696 p.

[29] В.В. Плавский, М.А. Шамсутдинов, Б.Н. Филиппов. ФММ 88, 22 (1999).

[30] Р.М. Вахитов, А.Р. Юмагузин. ФТТ 43, 65 (2001).

[31] Р.М. Вахитов, В.Е. Кучеров. ЖТФ 70, 67 (2000).

[32] B.A. Lilley. Phil. Mag. 41, 792 (1950).

[33] Р.М. Вахитов, Е.Б. Магадеев. ФММ 115, 306 (2014).

[34] I. Nistor, C. Holthaus, S. Tkachuk. J. Appl. Phys. 101, 09c526 (2007).

Редактор К.В. Емцев 